\title{
Crisis Celíaca: Una Grave Emergencia Pediátrica
}

\author{
Dr. Jorge Alvarez L.' ${ }^{1}$, Dra. Carmen Gutiérrez T. ${ }^{2}$, Dr. Ernesto Guiraldes C. ${ }^{2}$
}

\author{
The Celiac Crisis: A Pediatric Emergency
}

Celiac crisis, a life-threatening complication in children with celiac disease, has been considered a rarity by contemporaxy authors. Dur experience with this condition prompted this review of eight patients seen in our hospital over a period of six year. All them apeared with severe watery diarrhoea, accompanied by yomiting, dehydration, acidosis, hypoproteinemia, hypokalemia and progression to shock. The crisis was usually precipitated by infection and dietary indiscretion. Mean age of the group was 3.3 years. Chronic mainutrition and very low socioeconomic status were prominent features in the patients. Onty two children had been diagnosed as celiacs prior to the crisis; in the other six the condition was suspected on the basis of the typical clinical findings and confirmed later on by the criteria recommended by the European Society of Pediatric Gastroenterology. Initial therapy consisted in plasma infusions and rapid correction of the metabolic abnormalities. All patients were given a disaccharidefree, gluten-free diet and six required broad-spectrum antibiotics. Corticosteroids were administered to six patients who failed to respond to these measures. All showed a striking improvement after this therapy. Celiac crisis should be suspected in all chronically malnourished patients with antecedents and, or clinical features suggestive of a malabsorptive state and who present with an unusually severe watery diarrhoea leading rapidly to profound dehydration and acidosis.

(Key words: Celiac Crisis. Celiac Disease. Chronic diarrhoea Complications. Dietary indiscretion. Dehydration. Metabolic Disturbances. Shock).

La emergencia médica más seria que se puede presentar en un niño celiaco es la llamada "crisis

1. Becatio, Servicio de Pediatria, Hospital Luis Calvo Mackenna, Santingo.

2. Unidad de Gastroenterología, Hospital Luis Calvo Mackenna, Santiago. celíaca", la cual tiene una alta letalidad si no es abordada pronta y vigorosamente ${ }^{1-4}$. Este término se aplica a la descompensación de una enfermedad celíaca, con el desencadenamiento de diarrea severa, hiperemesis, deshidratación, acidosis, importante pérdida de peso y rápida 
progresión hacia la insuticiencia circulatoria. Habitualmente se produce en pacientes con hipoproteinemia marcada y escasez corporal, igualmente severa, de potasio, calcio y magnesio ${ }^{1.4}$.

La transgresión masiva, subrepticia o involuntaria, del régimen sir gluten es una condición indispensable para la precipitación de la crisis celíaca, y la infección, enteral o parenteral, es también un factor casi universal ${ }^{1}{ }^{4}$. En algunos pacientes la emergencia se confunde con una afección quirúrgica abdominal debido a la frecuente presencia de íleo paralítico. En estas circunstancias, una laparatomía exploradora pue. de ser fatal.

En las últimas décadas se ha publicado muy poco sobre crisis celíaca. Con los avances en el diagnóstico y tratamiento de la enfermedad celíaca, y la creación de una conciencia más amplia de la enfermedad entre la comunidad mèdica, los pacientes están menos expuestos a sufrir las graves consecuencias que eran de regla en épocas pasadas $y$, consecuentemente, la mortalidad se ha reducido drásticamente ${ }^{1}$.

A pesar de los progresos aludidos, aún subsisten numerosos problemas en la creciente población de pacientes celiacos bajo nuestro cuidado, que afectan principalmente a aquellos provenientes de los estratos socioeconómicos y culturales más bajos.

En la última hemos asistido a 8 pacientes con crísis celíaca, cuyos hechos clínicos y de laboratorio más relevantes, así como su respuesta al tratamiento adoptado, presentamos a continua. ción.

\section{PACIENTES Y METODOS}

Se revisaron las historias clínicas de 8 pacientes atendidos en el Hospital Luis Calvo Mackenna entre los años 1974 y 1980,5 mujeres y 3 varones, promedio de 3,3 años (1,2 a 8 años) de edad. Todos tenían una historia de diarrea crónica antes del ingreso (promedio 29,6 meses; rango: 4 meses a 7 años) y 5 provenian de áreas rurales (Tabla 1). En la mayoria de las condiciones socioeconómicas eran de extrema pobreza. Sólo dos de los pacientes eran celiacos conocidos que habían sido estudiados previamente con biopsia duodenal; los otros 6 nunca habían sido referidos para su estudio, consultando al hospital primariamente por la crișis. Posteriormente, todos ellos fueron estudiados apropiadamente, confirmándose la enfermedad de acuerdo con los criterios propuestos por la Sociedad Europea de Gastro. enterología Pediátrica ${ }^{5}$ que nuestro grupo utiliza en el diagnóstico de esta enfermedad ${ }^{7}$. Según este criterio, para hacer el diagnóstico de enfermedad celíaca, se requiere una biopsia inicial, una segunda que muestre mejoría histológica después de cierto ticmpo de tratamiento con régimen exento de gluten y una tercera que documente recidiva histológica al reintroducir gluten a la dieta ${ }^{5-7}$. Dadas las pésimas condiciones de nuestros pacientes al ingresar y por haberse usado en ellos corticoides, cuyo efecto reparador sobre la mucosa intestinal es muy rápido ${ }^{8-9}$, la biopsia inicial no pudo ser hecha en la mayoria de los niños. Sin embargo se hicieron las dos biopsias sigujentes $y$ en cada caso se produjo recaída histológica al reincorporar el gluten a la dieta años después de la crisis celíaca que motivó el ingreso.

\section{RESULTADOS}

La diarrea que motivó la hospitalización de los pacientes se caracterizó por gran número de evacuaciones líquidas abundantes, que originaron una intensa deshidratación (superior en todos los casos al $10 \%$ del peso corporal), con acidosis

Tabla 1.

Datos Generales de los Pacientes

\begin{tabular}{llcccc}
\hline Paciente & Sexo & $\begin{array}{c}\text { Edad } \\
\text { ingreso } \\
\text { (años) }\end{array}$ & $\begin{array}{c}\text { Area } \\
\text { Procedencia }\end{array}$ & $\begin{array}{c}\text { Duración } \\
\text { previa de la } \\
\text { diarsea } \\
\text { crónica } \\
\text { (aj̃os) }\end{array}$ & $\begin{array}{c}\text { Biopsia } \\
\text { dúodenal } \\
\text { previa }\end{array}$ \\
\hline 1. J.U.M. & Fem. & 6.5 & Rural & 5 & Sí \\
2. R.Q.M. & Fem. & 3.6 & Urbana & 3.2 & No \\
3. J.C.S. & Fem. & 1.6 & Urbana & 1 & No \\
4. M.D.V. & Fem. & 1.2 & Rural & 0.3 & No. \\
5.P.C.D. & Masc. & 1.7 & Rural & 0.6 & No \\
6. H.V.M. & Masc. & 2.5 & Urbana & 2 & No \\
7. E.Z.Z. & Fem. & 8.0 & Rutal & 7 & Sí \\
8. M.S.V. & Fem. & 1.7 & Rural & 0.3 & No \\
\hline
\end{tabular}


metabólica (Tabla 2). En siete niños hubo ade. más hiperemesis; en tres alteraciones de la conciencia en el ingreso, tres tenían fiebre $y$ seis edema de distinta magnitud, asociddocon hipo. albuminemia, Cuatro pacientes presentaban signos clínicos y radiológicos compatibles con íleo intestinal y concomitantemente, hipokalemia.

Tabla 2.

Hallazgos Clínicos más Importantes

Diarrea severa
Vómitos
Deshidratación severa $>10 \%$
Edema
lleo Intestinal
Eiebre
Alteraciones de conciencia

Una vez recuperados de la deshidratación, siete de los 8 pacientes estaban por debajo del percentil 5 de peso/edad (Tablas NCHS) y seis por debajo del percentil 5 de peso/talla; cinco de los 8 se encontraban bajo del percentil 10 de talla/edad y tres levemente por encima.

Las alteraciones bioquímicas más constantes fueron acidosis metabólica, hipokalenia, hipocalcemia y anemia. Las cifras de albúmina sérica alcanzaron en algunos casos, niveles extremadamente bajos, llegándose en un paciente a $17 \mathrm{~g} / \mathrm{l}$. El promedio del grupo fue de $22 \mathrm{~g} / 1$. El caroteno sërico, un elemento indirecto de evaluación de la magnitud de la malabsorción intestina ${ }^{6}$, fue en promedio de sólo $11.5 \mu \mathrm{g} / \mathrm{dl}$, en el grupo, siendo todos los valores individuales anomalmente bajos y aún no detectables en un niño (Tabla 3).

Tabla 3.

Hallazgos de Labotatorio más Importantes

\author{
Acidosis me tabólica \\ Hipokalemia \\ Hipoalbuminemia \\ Anemia \\ Hipocalcemía \\ Hipocarotenemia
}

(Promedio: $11,5 \mu \mathrm{g} / \mathrm{dl}$ )

En cinco pacientes se comprobó alguna infección al ingresar al hospital $y$ en 4 ocurrieron infecciones intrahospitalarias. En seis de los 8 pacientes se encontraron signos hematológicos sugerentes de infección (Tabla 4). En seis niffos fue necesario usar uno o más antibióticos de amplio espectro por las razones mencionadas.

La rehidratación por vía endovenosa, con corrección del contenido de electrolitos, debió ser
Tabla 4.

Infecciones demostradas y hallazgos hematológicos sugetentes de infección.

Infecciones en contradas al ingreso:

Salmonella B + E. coli enteropatógena

$: 1$

Giordia lamblia

Otitjs media aguda

$: 3$

Infecciones intrahospitalarias:

Bronconeumonia

Varicela

\section{Alteraciones hema tológicas:}

Leucocitosis con desviación izquierda

Neutrofillia

Neutropenia

vigorosa, para compensar la intensa deshidratación, en todos los pacientes. Todos los casos requirieron por lo menos una transfusión de plasma, en tres pacientes fue necesario emplear un número mayor.

El periodo de permanencia en el hospital fue prolongado (promedio 46 dias), lo que refleja más que nada el tiempo empleado en establecer el diagnóstico correcto, y las dificultades para conseguir la estabilización clínica de los pacientes. Todos fueron tratados con un régimen libre de gluten y sin disacáridos. El suministro de disacáridos en la dieta fue reestablecido gradualmente y sin problemas, después de un tiempo.

Debido a las malas condiciones clínicas en que se encontraban los pacientes, se decidió usar corticosteroides en 6 , en quienes la diarrea, los vómitos y las complicaciones metabólicas, se mostraban refractarios. Se empleó hidrocortisona en 4 niños, betametasona en uno y prednisona en otro, coincidiendo con lo cual se observó disminución significativa de las pérdidas fecales y la hiperemesis, notable mejoría del ánimo y del apetito, cambios que ocurrieron entre 24 y 72 horas después de iniciar el suministro de los corticosteroides.

En uno de los pacientes la crisis celíaca se complicó con una nefropatía hipokalémica, que produjo poliuria e hipostenuria mantenidas y requirió del uso de potasio por un periodo prolongado, obteniéndose la mejoria clínica del mes de tratamiento.

\section{DISCUSION}

Nuestros ocho pacientes caracterizaron por ingresar al hospital con diarrea extremadamente grave, a una edad en la cual es inusual sufrir complicaciones tan severas de la diarrea aguda. 
La desnutrición con componente agudo y crónico, fue otra característica del grupo, siendo en 6 de ellos de tipo pluricarencial. En todos había una escasez de proteinas del plasma, minerales y vitaminas, lo cual indicaba que su desnutrición era de origen complejo - con toda seguridad una malabsorción intestinal- - y de larga duración. En dos se conocía la existencia de enfermedad celíaca y ambos estaban infringiendo el régimen cuando desarrollaron la crisis celíaca, por lo que no representaron, en general, problemas para el diagnóstico. Sin embargo en los restantes, no habia antecedentes de que dicho diagnóstico hubiera sido documentado --ni siquiera considerado- en el pasado. Su historia clínica era pobre e imprecisa, hecho claramente determinado por la marginalidad (socioeconómica, cultural y geográfica) en que vivían. De modo que la probabilidad de que estuvieran sufriendo una crisis celiaca debiô surgir más bien de deducciones clínicas que de hechos establecidos.

Los pacientes que desarrollaban una crisis celíaca han sido, en general, descuidados por sus familias, por ignorancia, extrema pobreza, o franca irrresponsabilidad. Esto explica que lleguen en condiciones deplorables, con una diarrea crónica que puede haber durado años $y$, los celíacos conocidos, infringiendo abiertamente el régimen sin gluten. Ha sido nuestra experiencia, y la de otros grupos, que estos pacientes cambian permanentemente de hospital para no verse enfrentado a amonestaciones por parte de los médicos tratantes, por estar constantemente infringiendo el régimen terapéutico. Ha sucedido que, por temor a esta censura, los padres oculten deliberadamente los antecedentes celiacos del niño, privando a los médicos de información crucial para su manejo. Hemos conocido casos con historia clínica típica de enfermedad celíaca, que han sido traidos moribundos al hospital por sus padres, para fallecer luego en shock, descubriéndose posteriormente que habían sido estudiados en otros centros, con cuyas indicaciones $y$ seguimiento no cumplían.

En una de nuestras pacientes, celíaca conocida, la crisis se desencadenó cuando sus padres se separaron violentamente y el padre, como una manera de provocar daños a su cónyuge, arrebató a la niña de su lado y la alimentó deliberadamente con alimentos ricos en gluten. Afortunadamente pudo ser llevada a tiempo al hospital, donde sus antecedentes clínicos estaban consignados en la ficha.

Por todas las razones comentadas, no debe esperarse la historia clínica completa del pa- ciente para sospechar que existe una crisis celíaca y comenzar el tratamiento inmediato y vigoroso de esta grave emergencia médica. De acuerdo con nuestra experiencia y la de otros grupos ${ }^{4}$. creemos que toda diarrea grave en un niño que ha dejado atrás la edad de lactante, con evidencias claras de desnutrición ( $y$ sospecha fundada de malabsorción), debe tratarse como una crisis celíaca sin dilación. La rápida progresión hacia el shock hipovolémico exige la adopción de medidas urgentes, y de un manejo posterior muy cuidadoso, para salvar la vida de estos niños.

El uso de corticoides en esta situación es una medida salvadora, respaldada por la práctica clínica de varios grupos ${ }^{1-4}$. En nuestros casos, tuvo resultados dramáticos al revertir prontamente las graves alteraciones clinicas. Probablemente los esteroides actúan por varios mecanismos. Es sabido que son capaces de producir recuperación de la mucosa intestinal en pacientes celíacos, "in vivo" -9 e "in vitro" 10 ; los mecanismos moleculares de este efecto no son bien conocidos pero: posiblemente incluyan un efecto estabilizador de la membrana de los lisosomas de las células epiteliales, previniendo la liberación de enzimas hidrolíticas dentro de la célula ${ }^{11}$, y por otro lado, una inhibición de los efectos del gluten en la mucosa intestinal, mediante una acción sobre los diversos elementos del sistema inmu$n^{12}$. Los esteroides tienen además un conocido efecto antiinflamatorio $y$, en una enfermedad con un componente inmunológico, como es el caso de la enteropatía por gluten, pueden actuar en diferentes sitios. Por otra parte, son capaces de inducir algunas enzimas, $y$ en el intestino estimulan la actividad de la ATPasa dependiente de $\mathrm{Na}^{+}$y $\mathrm{K}^{+}$, pudiendo revertir procesos hipersecretorios $^{13}$. Adicionalmente, favoreciendo la reabsorción de sodio en el túbulo distal, llevan a una expansión del volumen extracelular, fuertemente deprimido en la crisis celíaca. Por último, en pacientes tan severamente comprometidos como los celíacos descompensados, puede presumirse cierto grado de insuficiencia suprarrenal $^{14}$, situación en la cual los corticosteroides resultarían claramente beneficiosos.

La duración del tratamiento esteroidal debería ser la estrictamente necesaria, disminuyéndose gradualmente la dosis del fármaco ${ }^{1}$. Los inconvenientes potenciales son compensados por su gran actividad terapéutica. El empleo de antibióticos neutraliza la posibilidad de que enmascaren $o$ exacerben una infección ${ }^{1}$.

Los mecanismos precisos que precipitan una crisis celíaca no se conocen bien. Sin embargo, 
son requisitos el consumo irrestricto de gluten, la hipoproteinemia, hipokalemia $y$, por lo general, una infección intestinal o extraintestinal. Los pacientes celíacos más pobres y descuidados son los más vulnerables y en ellos es probable que la insuficiente ingestión de alimentos, sumada a contaminación del intestino delgado y escasez crónica de nutrientes se potencien, para provocar la descompensación. Larrain ${ }^{4}$ ha comentado estos aspectos y ha enfatizado el posible rol deletéreo de una flora intestinal anormal ${ }^{15}$ sobre la mucosa, ya severamente dañada, del paciente celíaco.

Buscando una hipótesis unificadora es posible suponer que en un paciente con escasez de potasio, la menor motilidad intestinal resultante, pudiese favorecer la pululación bacteriana en el lumen, la que sería más marcada mientras mayor sea la exposición del paciente a contaminación ambiental del agua y alimentos. Este fenómeno se acentuaría con el uso de opiáceos y anticolinérgicos que, en algunos casos han servido de factor precipitante de la crisis celíaca ${ }^{1}$. pudiendo haber actuado a través de un mecanismo similar al megacolon tóxico de la colitis ulcerosa $^{1}$. La presencia de una flora cualitativa $y$ cuantitativamente anormal puede provocar varios fenómenos en el intestino delgado, pero hay dos que pueden revestir particular importancia: la absorción de endotoxinas a través de una pared desvitalizada con permeabilidad aumentada y la producción de enterotoxinas con acción secretora. que contribuirian a amplificar las perdidas de agua y electrolitos a través de una mucosa que ya se halla en un estado secretor basal, como presumible resultado del predominio relativo de las criptas sobre las vellosidades ${ }^{14}$. En tales condiciones, una infección enteral agregada, tendría efectos devastadores sobre el precario equilibrio inestable del paciente.

La hipolbuminemia, resultante de la acción combinada de una pobre ingestión de alimentos. de malabsorción de proteinas y de la pérdida intestinal de ellas por exudación, se agrava ante la presencia de infección, por el hipercatabolismo que esta produce. La hipoproteinemia severa favorece la aparición de edema asociado con una relativa reducción del volumen plasmático. En tales circunstancias, la imposicion de factores que disminuyan aún más la volemia, como un estado hipersecretor del intestino, desestabiliza aceleradamente la homeostasis hidroelectrolítica del paciente.

Junto con el uso de expandidores de volumen (plasma $y$ soluciones hidroelectrolíticas), y de corticoides, existen otras medidas indispensables en el manejo del paciente en crisis celiaca. La realimentación con fórmulas sin disacáridos se fundamenta en la existencia de una importante reducción de la actividad de las disacaridasas en la mucosa intestinal intensamente daftada. En estos casos la recuperación de la capacidad de hidrolizar azúcares $\mathrm{y}$, probablemente, de transportar con eficiencia los monosacáridos, parece ser lenta, y debe vigilarse con cautela. También es necesario aportar vitaminas, potasio, calcio y magnesio, para compensar la severa escasez de ellos $1-2-4$, de otro modo puede ocurrir, durante el período de recuperación de la crisis celíaca, complicaciones como la nefropatía hipokalémica y la tetania ${ }^{4}$. Esto último facilitado por varios factores que han sido analizados recientemente 4 .

Por último, el paciente que se ha recuperado de una crisis celíaca debe ser controlado rigurosamente. Además de la labor del médico, es necesa. ria la participación de la nutricionista y la asistencia social. Si no se prestá especial atención a la indole multifactorial del problema, se corre et resgo de que el paciente sufra nuevamente graves complicaciones.

\section{REFERENCIAS}

1. Lloyd-Still, J.D., Grand, R.J., Khaw, K.T. y Shwachman. $H .:$ The use of corticosteroids in celiac ctisis. J. Pediałr. B1: 1074, 1972.

2. Andorson, C.M. y Burke, V.: Coeliac Disease En: "Puediatric Gastroenterology" Eds. C.M. Anderson y V. Burke, Blackwel Scientific Publications, $\mathrm{Ox}$ ford, Capitulo 6, 1975.

3. C.C. Roy, A. Silverman y F.J. Cozzetto, C.V. Mosby Co. Sf. Louis: Pediatric Clinical Gastroenterology. Capítulo IV, 1975

4. Larrain, F.: Crisis celiaca, Pediatría (Santiago) 26: 34. 1983.

S. Meeuwisse, G.W.: Diagnostic criteria in coeliac disease. Acta Pacdiatr. Scand. 59: 461, 1970.

6. Guiraldes, E., Gutiemez, C. Rebolledo, L. y cols.: El examen del caroteno sérico en la diarrea crónica de la infancia. Rev. Chil. Pediatr. 45: 409, 1974.

7. Guiraldes, E., Gutiérrez, C. y Latorre, J.: La diarrea crónica en el niño. En: "Avances en Gastroenterolog ja". Tomo II. Eds. V. Valdivieso, R. Armas, y C. Quintana. Soc. Chilena de Gastroenterología, pp 252-378, 1979 .

8. Walt, A.J., Dougies, A.P. Both. C.C., y Pearse, A.G.E.: Response of the jejunal mucosa in adult celiac disease to osal prednisone. Gut $11: 7,1970$.

9. Danis, O., Larrain, F. y Mirkin, D.: Tratamiento esteroidal de la enfermedad celíaca del nin̄o. Rev Chil. de Pediatr. 45: 421, 1974 .

10. Falchuk, Z.M., Gebhard, R.L., Sessons, C. y Stro. ber, $W$.: An in vitro model of gluten-sensitive enterophaty. Effect of gliadin on intestinal epithelial cells of patients with gluten-sensitive enteropathy in organ culture. J. Clin. Invest. 54: 487, 1974. 
11. Riecken, E.O. Stewart, J.S., Boorh, C.C., y Pearse, G.E. A histochemical study on the role of lysosomal enzymes in idiopathic steatorthoea before and during a gluten-free diet. Gut 7: 317, 1966 .

12. Editorial. Response of gluten-sensitive enteropathy to corticoids. Nutr. Rev. 39: 132, 1981.

13. Chamey, A.N. y Donowitz, M.: Prevention and reversal of cholera enterotoxin-induced intestinal secretion by methylprednisolone induction of $\mathrm{Na}+$ K+ - ATPase. J. Clin. Invest. 57: 1590, 1978.

14. Trier, J.S.: Celiac Sprue Disease. En: "Gastrointestinal Disease". Eds. M.H. Sleisenger y J.S. Fordtran. W.B. Saunders Co. Vol. II. Capítulo 60, 1978

15. Lloyd-Still, J.D. y Shwachmon, H.: Duodenal microflora: A prospective study in pediatric gastrointestinal disorders. Dig. Dis. Sci. 20: 78, 1975. 